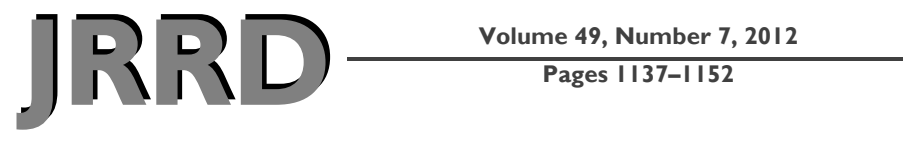

\title{
Preliminary framework for Familiar Auditory Sensory Training (FAST) provided during coma recovery
}

\begin{abstract}
Theresa Louise-Bender Pape, DrPH, MA, CCC-SLP/L; ${ }^{1-3^{*}}$ Joshua M. Rosenow, MD, FACS; $^{4-5}$ Brett Harton, BS; $^{1}$ Vijaya Patil, MD; ${ }^{6}$ Ann Guernon, MS; ${ }^{1,3}$ Todd Parrish, PhD; ${ }^{7}$ Kathleen Froehlich, OTR $/$; $^{8}$ Catherine Burress, DPT; ${ }^{8}$ Shane McNamee, MD; ${ }^{9}$ Amy A. Herrold, PhD; ${ }^{1}$ Bessie Weiss, RN, MS, CCNS, CCRN, CNRN; ${ }^{5}$ Xue Wang, PhD $^{7}$ ${ }^{1}$ Department of Veterans Affairs (VA) Center for Management of Complex Chronic Care, Center of Excellence, and Research Service, Edward Hines Jr VA Hospital, Hines, $I L ;{ }^{2}$ Department of Physical Medicine and Rehabilitation, Northwestern University Feinberg School of Medicine, Chicago, IL; ${ }^{3}$ Marianjoy Rehabilitation Hospital, Wheaton, IL; ${ }^{4}$ Department of Neurological Surgery, Northwestern University Feinberg School of Medicine, Chicago, IL; ${ }^{5}$ Northwestern Memorial Hospital, Chicago, IL; ${ }^{6}$ Neurology, Edward Hines Jr. VA Hospital, Hines, IL; and Department of Neurology, Loyola University Chicago, Stritch School of Medicine, Maywood, IL; ${ }^{7}$ Department of Radiology, Northwestern University Feinberg School of Medicine, Chicago, IL; ${ }^{8}$ Brain Injury Program, The Rehabilitation Institute of Chicago, Chicago, IL; ${ }^{9}$ Physical Medicine and Rehabilitation Service, Hunter Holmes McGuire VA Medical Center, Richmond, VA
\end{abstract}

\begin{abstract}
Since there remains a need to examine the nature of the neural effect and therapeutic efficacy/effectiveness of sensory stimulation provided to persons in states of seriously impaired consciousness, a passive sensory stimulation intervention, referred to as the Familiar Auditory Sensory Training (FAST) protocol, was developed for examination in an ongoing, double-blind, randomized clinical trial (RCT). The FAST protocol is described in this article according to the preliminary framework, which is a synthesis of knowledge regarding principles of plasticity and capabilities of the human brain to automatically and covertly process sensory input. Feasibility issues considered during the development of the intervention are also described. To enable replication of this intervention, we describe procedures to create the intervention and lessons learned regarding the creation process. The potential effect of the intervention is illustrated using functional brain imaging of nondisabled subjects. This illustration also demonstrates the relevance of the rationale for designing the FAST protocol. To put the intervention within the context of the scientific development process, the article culminates with a description of the study design for the ongoing RCT examining the efficacy of the FAST protocol.
\end{abstract}

Key words: auditory stimulation, coma, disordered or impaired consciousness, minimally conscious state, plasticity, rehabilitation, story, traumatic brain injury, treatment, vegetative state.
Clinical Trial Registration: ClinicalTrials.gov; NCT00557076, "The Efficacy of Familiar Voice Stimulation During Coma Recovery"; http://clinicaltrials.gov.

\section{INTRODUCTION}

Some survivors of severe traumatic brain injury (TBI) will recover full consciousness, and others will remain in

\footnotetext{
Abbreviations: BAEP = brainstem auditory evoked potential, $\mathrm{CD}=$ compact disc, CNS = central nervous system, DOCS = Disorders of Consciousness Scale, FAST $=$ Familiar Auditory Sensory Training, fMRI = functional magnetic resonance imaging, LTM = long-term memory, MCS = minimally conscious state, $\mathrm{MMN}=$ mismatched negativity, $\mathrm{PET}=$ positron emission tomography, RCT = randomized clinical trial, STM = shortterm memory, $\mathrm{TBI}=$ traumatic brain injury, VA = Department of Veterans Affairs, VS = vegetative state.

*Address all correspondence to Theresa Louise-Bender Pape, DrPH, MA, CCC-SLP/L; Edward Hines Jr VA Hospital, PO Box 5000 (M/C 151H), Building 1, Hines, IL 60141. Email: Theresa.Pape@va.gov http://dx.doi.org/10.1682/JRRD.2011.08.0154
} 
states of seriously impaired consciousness for protracted periods of time [1-7]. Regardless of whether severe TBI survivors do or do not recover full consciousness, severe TBI is a catastrophic event involving significant cognitive and physical impairments. Increased survival rates; advances in life sustaining technology; and the complex, chronic nature of severe TBI have, collectively, raised awareness of the need for interventions to minimize the disabling effects of severe TBI. In response to this need, the experimental Familiar Auditory Sensory Training (FAST) protocol was developed for examination in a double-blind randomized clinical trial (RCT). We chose to develop a sensory stimulation treatment protocol because, despite the scarcity of evidence regarding therapeutic efficacy, sensory stimulation is customarily provided to patients in states of seriously impaired consciousness [8-13].

The primary objective of the ongoing RCT (approved by the human subjects institutional review board for each participating site) is to determine the therapeutic efficacy of the FAST protocol. The purpose of this article is to describe the FAST protocol according to the underlying conceptual framework.

\section{Description of Experimental Intervention: FAST Protocol}

The intervention is the FAST protocol, in which a research participant listens to a customized compact disc (CD) of digital recordings of a familiar voice first calling the subject's name aloud and then telling a story about a memorable event from the "storyteller's" perspective. The storytellers are selected by the research participant's legally authorized representative. Persons eligible to be storytellers are those familiar to the research participant. The term "familiar" means that the persons (1) knew the participant for at least 1 year prior to injury, (2) experienced/participated in events with the research participant, and (3) interacted with the participant on a routine basis for at least 1 year prior to the severe TBI. Up to three different familiar people (e.g., spouse, sister, friend, parent, work colleague, or classmate) can serve as storytellers.

Each story starts with the storyteller calling the research participant's name aloud three times. The name should be that used by the storyteller to refer to the research participant, and as such, it can be a nickname if the storyteller verbally referred to the participant by this nickname on a routine and common basis (1 year prior to injury). Each story must reflect the storyteller's perspective and recall of a memorable and specific event (e.g., ski trip, wedding, or birth of a child). Only events that were jointly engaged in by the storyteller and research participant are eligible to be stories included in the FAST protocol. All events must also have occurred at least 1 year prior to the date of the participant's severe TBI. Four of the eight events must involve happy memories, and the other four events must involve sad, negative, or neutral memories. The emotions associated with each memory are those of the research participant. The storytellers identify the emotions that they think the participant attributes to the event. Stories cannot involve fearful memories or describe events likely to invoke fear in the research participant. Each story describes a different event, but if there is more than one storyteller, the stories may describe a similar event from each storyteller's own perspective.

A minimum of eight stories are obtained from the one to three selected storytellers. The goal is for each story to last 5 minutes, but if some stories are shorter, more than eight stories will be needed because the minimum duration for story material is 40 minutes. The total duration of the FAST protocol is 1,680 minutes of familiar auditory sensory material, which is provided daily over 6 weeks. Each day of treatment is composed of 40 minutes of stories provided four times daily, in 10-minute segments at least 2 hours apart.

\section{Conceptual Framework for Developing Experimental Intervention}

The conceptual framework underlying the FAST protocol is a synthesis of knowledge regarding principles of neural plasticity, largely experience-dependent neural plasticity, and knowledge regarding the abilities of the human brain. We theorized that an intervention that applies certain principles of plasticity while leveraging the capabilities of the brain to automatically and covertly process sensory input would result in improved neurobehavioral functioning.

There are recent reviews in the literature summarizing the evidence of experience-dependent plasticity, and the majority of the principles that we translated to develop the experimental intervention are eloquently discussed in two reviews; one by Kleim and Jones in 2008 [14] and one by Ludlow et al. in 2008 [15]. In lieu of comprehensively reviewing this evidence, we describe each principle in relationship to how it influenced development of the experimental intervention. We also provide salient examples from the literature to illustrate each principle.

The intervention leverages evidence related to known abilities of the uninjured and injured human brain, and this evidence is derived largely from the body of neurosciences literature related to automatic and covert neural 
processing. Since a comprehensive synthesis of this evidence is beyond the scope of this article, we describe these abilities and then illustrate each with examples from the literature.

\section{Applying Principles of Experience-Dependent Neural Plasticity}

The principles of plasticity collectively and in combination with knowledge of the abilities of the human brain provide the basis for the experimental intervention. The principles of plasticity, in isolation, do not sufficiently form a framework for the FAST protocol, but to enhance clarity, we describe each principle separately in this section.

The first principle is that relearning promotes plasticity because it creates functional neural connections. While this principle is based on ample evidence that the nervous system changes to support relearning [16], we also know that severe brain injury will change the way relearning will occur. Fortunately, there is a growing body of evidence from the basic sciences and clinical stroke literature that the neural processes supporting relearning alone and in combination with rehabilitation exercises can promote plasticity of the injured brain [17]. Since the evidence indicates that each patient with severe brain injury will have unique neural processes supporting relearning, the translation of this principle means that we should develop an intervention that could be customized to each patient and combined with other training tasks.

A principle closely aligned with relearning is "specificity," which means that the nature of the plasticity will be dictated by the nature of the training experience. To produce significant changes in patterns of neural activity and potentially brainstem-thalamo-cortical connectivity (disrupted after severe $\mathrm{TBI}$ ), the training experience must involve relearning of a skill or knowledge rather than mere use or exposure. A training experience that involves relearning involves a specific modality because it is thought that focusing on a specific modality changes "subsets of neural circuitry involved in more general function," thereby influencing the capacity to acquire other functions in nontrained modalities [14]. This principle was eloquently illustrated in a recent review of the literature regarding the distinct neurophysiological mechanisms subserving training-induced plastic changes within auditory pathways (e.g., learning to discriminate environmental sounds) [18]. Translation of this principle to address our goal, of developing an intervention to improve neurobehavioral functioning in general, indicated that we should identify a sensory task that will dictate higher-level cortical activations and processing and that will reference and possibly engage subsets of neural circuits. As explained in more detail later in this article, this is one reason why we chose familiarity, particularly explicit episodic-related familiarity, for our sensory training task.

Two closely related principles are "use it or lose it" and "use it and improve it" [14-15]. The former indicates that neural circuits will degrade if not engaged in task performance for extended periods of time and that the failure to use specific brain functions will lead to skill degradation. This was first demonstrated in the 1960s when kittens were deprived of light, and after deprivation, the number of neurons in the visual cortex responding to light was reduced [19]. The rationale for providing sensory stimulation to persons in states of seriously impaired consciousness [12-13,20-21] is based largely on the "use it or lose it" principle.

"Use it and improve it" indicates that training experiences that use specific brain functions can enhance those functions [15]. Some of the first research supporting this principle was published by Nudo et al. in 1990, who demonstrated that intracortical microstimulation of adult rats on the area of the motor cortex that represents caudal and rostral forelimb movements significantly expanded this cortical representation [22]. Later work demonstrated that the rat motor cortex reorganized after motor movements improved following motor skill training tasks [23]. Applying this principle indicated to us that optimal sensory input is input that targets specific neural functions or circuits to reference and perhaps engage.

Changing intensity and repetition are two closely related principles of experience-dependent plasticity. Changes in neural substrates via changing intensity and repetition have been demonstrated in several bodies of literature, including the highly repetitive multimodal training undertaken by professional musicians [24]. Professional musical training illustrates that neural changes may not become consolidated until after skill mastery and that use of multiple modalities during training increases or changes the intensity of training without, necessarily, increasing the repetition of identical exercises [25]. That is, the evidence indicates that use of multiple modalities to engage or reference a common subcircuit will increase or change the intensity of referencing or engaging of the common subcircuit [24]. Therefore, intensity can be increased or changed via multiple repetitions of identical tasks [26] and via use of multiple modalities targeting the same subcircuits. 
Evidence derived from studies of constraint-induced language therapy techniques illustrates the use of intense repetition of highly similar exercises to improve function [26]. These techniques promote use of the impaired linguistic functions continuously without pause, and evidence indicates some success with this intense repetition [26-27]. We translated the principle of intense repetition cautiously because increasing intensity simply by increasing the number of repetitions of identical tasks can cause habituation and overuse, and this is particularly concerning for severe brain injury. The concept of muscle fatigue, related to performing too many of the same physical exercises, illustrates our concern. That is, there is evidence of maladaptive responses to highly intense motor treatment programs involving overuse of a muscle [28].

Some forms of plasticity require relearning of the knowledge or skill as well as continuous repetitive performance of the skill over time and, perhaps, with greater intensity, but for persons with a severe brain injury, central nervous system (CNS) metabolic fatigue and subcircuit fatigue must be considered when determining intensity and repetition. Application of the principles of changing intensity and repetition to develop our intervention meant, therefore, that we had to determine a daily "dose" of the experimental stimulation with a schedule of repetition that would not overly fatigue the patient. These principles indicated further that we had to develop multiple modality inputs that target common subcircuits, thereby changing intensity while avoiding intense repetition of identical sensory input.

The experience-dependent principle referred to as "saliency" is based on evidence indicating that there is a neural system that mediates saliency and, further, that engaging this system is critical to driving experiencedependent plasticity. While we are far from understanding the underlying neural mechanisms that modulate recovery by saliency, Kleim and Jones summarize ample evidence indicating that acetylcholine, emotion, motivation, and attention each contribute to saliency of training experiences [14]. There is also emerging evidence that the midbrain (superior colliculus) contributes to mediating saliency of sensory input [29]. Given the evidence collectively, we sought to leverage these contributors to saliency when developing the content of the intervention.

\section{Evidentiary Concepts of Human Brain's Capabilities}

In addition to applying the previously mentioned principles of neural plasticity, the FAST protocol was developed in accordance with evidence of the human brain's ability to (1) automatically respond to passive sensory input, (2) covertly process sensory information, (3) automatically respond to unexpected auditory stimuli, (4) retain new information on a short-term basis, (5) engage longterm memory (LTM) circuits, (6) distinguish between familiar and nonfamiliar events, and (7) orient to incoming information. These abilities are described separately and examples of investigations reported in the literature are provided to illustrate these abilities.

\section{Automatic and Covert Neural Processing of Passive Sensory Input}

There is evidence from investigations of a variety of patient populations indicating that human brain activity changes automatically in response to passive sensory input and, further, that neural processing can occur in a manner that is not detectable at a behavioral level or covertly. One study illustrating the concept of automatic responsiveness involved providing anesthetized patients undergoing cardiac surgery with auditory instructions regarding a specific memory task [30]. During the exposure to the auditory information, the patients exhibited automatic changes in auditory evoked potentials. A more pertinent example is a study conducted with five patients in a vegetative state (VS) from hypoxic events [31]. This report provided evidence of automatic changes in activation within the primary auditory cortex, as measured with auditory evoked potentials, while the patients were listening to clicking sounds.

The study of anesthetized cardiac patients also illustrates the concept of covert processing. Postanesthesia, the patients had significantly better implicit recall (i.e., primed recall of memory) relative to explicit recall (i.e., conscious recollection of memory) for the events they were instructed to remember (while anesthetized) [30]. Investigations of visuospatial neglect further illustrate the concept of covert processing; Marshall and Halligan, for example, reported evidence indicating that a patient with left visual neglect could not directly supervise visual attention to the left visual field, but could discriminate between visual pictures presented in the left visual field [32]. Marshall and Halligan's patient judged two pictures presented in the left visual field to be identical, but the pictures were actually different-one was a picture of a burning house and the other was a picture of the same house that was not burning. When asked to choose which house she preferred to live in, despite judging the pictures to be identical, the patient chose to live in the house that was not burning. 
There are case reports of persons in states of seriously impaired consciousness that also provide evidence illustrating the concept of covert processing [33-36]. Moritz et al., for example, reported evidence of cortical activation measured with functional magnetic resonance imaging (fMRI), in the absence of behavioral responses, to visual (blinking light), auditory (narrated text), and somatosensory (bilateral palm scratching) stimuli [36]. Detected activation was in expected regions of the cortex, suggesting covert processing of passive sensory information. Menon et al. used positron emission tomography (PET) to study covert processing for a patient who had become comatose after a febrile illness [35]. The patient did not demonstrate consistent motor or visual behavioral responses to passive sensory input. During the PET scan, the patient was exposed to photographs of familiar faces and to control photographs (i.e., same pictures but repixellated to remove structure), and the familiar pictures elicited significant cortical activation in logical areas (e.g., right fusiform gyrus).

\section{Automatic Neural Responses to Unexpected Auditory Stimuli}

Evidence regarding the brain's ability to covertly process passive sensory input and automatically detect change in input is particularly well established for the auditory system (e.g., emergency alert signals interrupting regular radio programming), and this evidence is derived largely from investigations of "mismatched negativity" (MMN) [37]. MMN is an index of the brain's response that is evoked when a deviant or unexpected auditory event is inserted into a series of repeated auditory events [38-39]. $\mathrm{MMN}$, an index of the brain's automatic response to detecting this unexpected change in routine auditory input, can be elicited by altering aspects of the auditory input including frequency, duration, intensity, sound location, silence in place of tone, or phoneme replacement. MMN is typically measured with electroencephalography or magnetoencephalography [38], but some studies also measure it with electrooculography [39-40]. MMN is calculated as the difference or mismatch between the brain's response to the repetitive sequence of auditory input and the brain's response to the deviant or unexpected auditory input [41]; the difference between responses or mismatch in responses equals MMN.

MMN studies of the auditory system indicate that automatic brain activity is elicited in the absence of supervised attention [40]. A pertinent example of this concept is a study in which electrophysiological recordings of comatose patients were completed within 48 hours of occurrence of a severe brain injury. The MMN signal was elicited in 4 of the 18 patients, and these 4 patients recovered full consciousness within 48 hours of the recordings, while the remaining 14 patients did not [42]. Another study examined MMN in patients who remained in a VS for at least 1 month postinjury. The amplitude and peak latency of the MMN signal were analyzed as a function of levels of consciousness [39]. Results from another MMN experiment with patients in VSs and minimally conscious states (MCSs) measured MMN in response to the subject's own name being called aloud. Reported findings indicated that the presence of MMN response significantly correlated with transition from VS to MCS [43]. While the authors of each of these MMN experiments theorized that the presence of MMN signaled recovery of the neural mechanisms necessary for information processing and/or recovery of consciousness [39,42-43], the presence of MMN illustrates that automatic detection of auditory change can be independent of supervised attention to auditory stimuli.

\section{Role of Short- and Long-Term Memory in Automatic Detection of Unexpected Auditory Stimuli}

MMN evidence indicates that the human brain's ability to automatically respond to unexpected auditory input is enabled, in part, by the ability to activate recent sensory input stored on a short-term basis (short-term memory [STM]) in the auditory cortex [44] and by the ability to activate previously established memory traces specific to familiar auditory elements encoded in neuronal assemblies or LTM circuits [40-41] that are distributed over sensory, motor, and association areas of the brain [16].

Neurocomputational studies analyzing the STM and LTM components of eliciting MMN [41] indicate that neuronal adaptation [45] and local inhibition [46] of cortical activity explain the brain's ability to detect change when a repeated unfamiliar sound changes in frequency. The brain's responses to changes in repeated familiar stimuli, however, are enabled by neuronal adaptation, local inhibition, and activation of LTM circuits that emerged in the cortex during the learning of these stimuli. Automatic neural responses observed when familiar auditory input is unexpectedly changed are much stronger, relative to unfamiliar auditory input, because LTM circuits are engaged [41]. In summary, activation of LTM circuits is not necessary for automatic detection of changes in unfamiliar auditory stimuli patterns, but it is necessary for automatic detection of changes in familiar auditory stimuli. 


\section{Processing of Familiar and Emotional Information}

Given that the brain's ability to engage an LTM trace from a familiar auditory prompt involves processing in higher-level association areas as well as sensory systems, it is not surprising that processing of familiar stimuli and emotional information also involve higher-level cortical processing. A seminal investigation by Imaizumi et al. [47] demonstrated, for the first time, that the neural processes responsible for speaker identification via vocal recognition are the same processes involved in familiar face recognition (i.e., fusiform face area and the bilateral temporal poles) [48]. Imaizumi et al. studied six healthy males who completed speaker and emotion identification tasks during a PET scan. Four speakers (two male and two female) spoke three examples each of eight nonaffective words with four different emotional tones-surprise, disgust, happiness, and anger. The six subjects guessed the identity of the speaker and guessed the expressed emotion. Reported findings indicate that familiar voice perception involves identification of prosody (e.g., pitch, tempo), and the right hemisphere plays a dominant role in prosody perception. We also know that the left auditory cortex is more involved in the processing of vocal (e.g., speech encoding) versus nonvocal (e.g., tones) information [49-50]. Imaizumi et al. demonstrated further that vocal identification of a speaker involves the bilateral temporal poles, right lingual gyrus, and left parahippocampus. Temporal pole involvement is thought to be related to recall of known phenomenon, and hippocampal involvement is thought to contribute to the reestablishment of connections between vocal representations and biological information stored in LTM.

Imaizumi et al.'s results also showed that identification of vocal emotion involved the cerebellum, left middle frontal gyrus, and right inferior frontal gyrus. Results from investigations of emotional disorders served to support Imaizumi et al.'s findings regarding the relationship between emotion identification and the cerebellum [5152]. The prefrontal cortices have also been found to be involved in identification of negative and positive vocal emotions [47]. The cerebellum is thought to be involved because affective stimuli induce subjective feelings corresponding to changes in gestures, facial expressions, and physiological indicators. Emotional identification, therefore, involves recall and autonomic activity driven by the cerebellum.

Additional investigations expanded upon Imaizumi et al.'s findings regarding the neural processes that enable familiar face, familiar voice, and emotion recognition [5354]. These later investigations indicate that, irrespective of sensory modality, familiarity processing is more specifically associated with increased activity in the posterior cingulate cortex, including the retrosplenial cortex, whereas the left frontal and right temporal poles are involved in the recognition of familiar voices. Neuropsychological studies specify further that the right parietal region plays a role in familiar voice identification or recognition, and that the bilateral temporal regions are involved in discrimination of unfamiliar voices [55-56].

\section{Brain Priming and Familiarity Processing}

Studies of neural responses to a subject's own name called aloud after anesthesia and during coma indicate that familiarity processing enhances the subsequent processing of novel information by capturing the comatose patient's attention and orienting (i.e., prefrontal activity) the patient to subsequent stimuli [57-59]. In addition to a priming effect, reported evidence indicates that the probability of eliciting more responses to auditory tones increased when tonal stimuli were coupled with familiar vocal stimuli such as the subject's own name. These findings are in accordance with several studies in which results indicate that calling the subject's own name aloud captures attention without directed or focused effort and disrupts ongoing cognitive activities [60-63]. In 2007, Di et al. reported evidence indicating that auditory cortex activation was elicited for five of seven VS patients when their name was called aloud by a familiar voice. While Di et al. did not report the use of a control task, they did report that four MCS patients demonstrated similar responses and two of the MCS patients had activation in higher-level association areas (i.e., middle temporal and superior temporal areas) [64].

\section{Summary of Preliminary Framework for FAST Protocol}

To leverage evidence of how brain priming enhances processing of subsequent stimuli, each story starts with the patient first being alerted to upcoming stimuli via hearing their name called aloud. The stories leverage preinjury memories of voices/prosody, people (i.e., syntactic language structure, pragmatics), and events (i.e., experiential/ episodic memory) that are happy, sad, and neutral and are encoded in LTM [65]. Rather than referencing or engaging STM circuits, enabled when auditory information is retained on a short-term basis in the auditory cortex, we 
sought to engage or reference LTM circuits because of STM deficits typical with severe TBI. Therefore, we sought to optimize the possibility of engaging or referencing well-established neural circuits because "exercising" these LTM circuits should promote higher-level cortical activation of neural processes related to LTM, familiarity, and auditory processing.

Stories describing specific events, rather than recalling other familiar information encoded in LTM (e.g., college major, career plans and aspirations, family genealogy), were selected to leverage knowledge related to the principle of specificity. That is, stories related to specific events will be richer because they are laden with details that can possibly reference multiple sensory modalities. Retelling of an event can include visual, tactile, olfactory, and auditory descriptions that help to bring the story "to life." Leveraging LTM circuits also allowed us to customize the intervention to each subject, thereby optimizing opportunities for engaging neural processes unique to each subject that must be enabled to promote relearning. We theorized that targeting an LTM circuit via multiple sensory modalities described verbally would possibly reference or engage a common subset of neural circuits involved in general neurobehavioral functioning.

Familiar, rather than nonfamiliar, voices were selected for the stories because of evidence of differential (i.e., more) neural activity to voice pattern recognition and contrasting pitch with complex auditory sequences within the primary auditory areas as well as downstream auditory association areas. This evidence of increased activation to familiar voices in higher-level cortical areas combined with evidence that engagement of LTM traces requires familiar stimuli indicated that familiar people, encoded in the participant's LTM, should be used as the storytellers.

Emotional content is included in the stories (i.e., happy, sad, and negative events) because emotion is an inherent parameter of the LTM trace and because passive emotion identification is also known to engage higher-level cortical processing and/or elicit unsupervised neural responses. Leveraging stories laden with emotions enhances motivation and attention to the sensory training experience, thereby optimizing the principle of saliency. To minimize the possibility of overloading the brain's ability to engage emotional processing mechanisms for patients in states of seriously impaired consciousness, we ensure that one or two of the eight stories are neutral in emotion.

To leverage knowledge of the roles of repetition, relearning, and referencing and/or engagement (activa- tion) of LTM traces, we repeat the intervention daily for 6 weeks. We chose to repeat the stimulation four times a day for 6 weeks because of the relationships between repetition and learning, but we also chose to play eight different stories to minimize the possibility of habituation and to leverage knowledge of how intensity can be changed when multiple tasks target the same LTM circuits. While the schedule of repetition was driven by practical considerations, such as working around scheduled therapy sessions and requiring the patient to be awake to listen to the CDs, the schedule of repetition was largely determined according to the evidentiary framework. That is, the stimuli needed to be repeated enough to maximize opportunities to systematically manipulate neural activity in a controlled manner to ultimately alter axonal impulses and interrupt or modulate ongoing neuronal activity so that LTM traces could be engaged or referenced [66-67]. Repeated passive exposure to familiar vocal stimulation, in the format of stories with largely emotional content describing events encoded in LTM, is used as the experimental sensory stimulation intervention because evidence indicates that the human brain can automatically (1) elicit covert neural processing, (2) elicit unsupervised neural responses, and (3) reference and/or engage LTM traces [68].

In summary, the underlying principle of the FAST protocol is that it is structured so as to be relevant, salient, and inherently recognizable by persons in states of seriously impaired consciousness. The auditory training is familiar to the patient because it is specifically designed to be experientially relevant and, thus, has the greatest chance of actively referencing and engaging neural circuits. The presumption is that plasticity operates under a principle of active engagement of the subject rather than passive stimulation of a subject presumed to be broadly receptive. The context-dependent stimulation, in which the context must take into account the relevancy of the stimulation for each participant, is key to optimizing the possibility of active engagement and emotional response.

\section{METHODS: FAST PROTOCOL-PROCEDURES FOR CREATING STORIES}

A clinical researcher (i.e., physical, occupational, or speech therapist) initiates a dialog with the three storytellers identified by the research participant's legal representative. 
The purpose of the initial dialog is to provide support and to facilitate creation of the stories. This dialog involves reviewing the FAST protocol with the storyteller and answering their initial questions. The researchers encourage storytellers to use multiple strategies to identify story topics, such as looking at photographs and videos and talking with other friends/family members. Recalling special occasions such as holidays, vacations, or birthdays has helped storytellers conjure up memories that can be used in the happy recordings, and recalling job losses, home remodeling projects, deaths in the family, or moving may be used in the sad and neutral recordings. Storytellers are encouraged to include sensory details (visual, olfactory, tactile, auditory), such as where the event took place; the people who were there; and all of the sights, sounds, and smells associated with that memory. The inclusion of these details "set[s] the sensory scene" for the story. Once the sensory scene is set, the storyteller is instructed to write their story and then time themselves at least once to ensure the duration of each story is adequate.

After the storyteller has practiced reading the story aloud with the clinical researchers, the story is then recorded using a handheld digital voice recorder. The recordings take place in a private room to make the storyteller comfortable and keep background noise to a minimum. The storytellers are instructed and coached by the clinical researchers to speak in their normal voice (volume, pitch) and to use normal speech patterns (prosody, tone, rate), without editing for specific grammar, syntax, or pronunciations that are not inherent to the storyteller.

Once the stories are digitally recorded, they are then edited for length and sound quality using a commercially available sound editing program. Stories longer than 5 minutes are edited down to be as close to 5 minutes as possible. Distracting background noises such as echoes, door slams, or other white noise are eliminated. Aspects of the storyteller's natural or normal speaking patterns (e.g., speech rate) are not edited. Once the stories have been edited for sound quality and content, the stories are all adjusted to the same volume level (using the same sound editing software), between 50 to $75 \mathrm{~dB}$. Prior to copying the soundedited stories onto CDs, we listen to the stories through headphones to make sure that the volume is at a comfortable listening level without abrupt increases or drops in volume levels. After creating the CDs, the sound levels are checked a third time with a sound level meter.

\section{RESULTS: LESSONS LEARNED}

While ideal scenarios have presented themselves to researchers on more than one occasion, there have also been unique challenges and lessons learned regarding the creation of the stories. An example of the ideal scenario is when one family quickly identified three people to complete recordings. One family member voluntarily took the lead to delegate and coordinate who would do each story and instructed each storyteller to contact the research team by a specific deadline. Prior to the delegated deadline, each storyteller had contacted the researchers and scheduled a recording session. Each storyteller had the stories written and timed prior to the scheduled session, ensuring a sufficient amount of content for a 5-minute story. Recording was completed with each of the storytellers in a single session that lasted approximately 30 minutes.

Since each patient and family presents a unique set of circumstances, creating and recording the stories is not always as straightforward as the preceding example. Issues that potential storytellers are dealing with may include the acute stages of coping with the traumatic event that caused the participant's injuries. This can result in difficulty focusing on creating and recording the stories. The stories involving negative memories may be especially challenging at this time, particularly if the stories raise unresolved issues.

Other challenging issues relate to the quality of the relationships between the storytellers, having enough content for stories, or motivation to generate the stories. One subject, for example, had family members identified to record stories quickly, but the relationships between the storytellers were strained. The research team took the lead, in this situation, to discuss the protocol with each storyteller individually and to coordinate who would do the happy versus sad and neutral stories. Despite being provided a story completion deadline, the psychological and emotional strain the storytellers' were experiencing delayed story completion. Some family members had not timed their stories prior to recording sessions, necessitating multiple and protracted sessions to allow revisions and additions. Another storyteller started with a story that involved a mutually experienced LTM, but then shifted into plans for the future as the main content of the story. This story was eliminated, and an additional story was obtained.

While each research participant's circle of family, friends, and/or significant others presents unique circumstances and issues, the one common theme is engagement. 
That is, the storytellers have reported feeling as though they have made a meaningful contribution to the participant's rehabilitation as well as a sense of control over a segment of the participant's medical rehabilitation program.

\section{DISCUSSION}

\section{Conceptualizing the Intervention: Feasibility Issues}

The FAST protocol was developed to address the need for an affordable sensory stimulation intervention that can be feasibly implemented in daily rehabilitation. Therefore, while conceptualizing the intervention, feasibility issues related to affordability and implementation by allied health clinicians and caregivers during and after acute rehabilitation were carefully considered. A further constraint imposed by the study design is the use of fMRI as one method to investigate mechanisms of neurobehavioral changes detected in the ongoing RCT.

We sought to develop an intervention that would be affordable for most patients, regardless of their healthcare insurance benefits. Affordability relates to both the device used to deliver the sensory input in a standard manner and also to the fact that the majority of patients receive unpaid long-term caregiving services from family members, significant others, and friends. Many devices used to deliver customized visual sensory information in a standard manner are either expensive, require computer programming, and/or require costly maintenance (e.g., visual laser chase technology, virtual reality). The auditory modality offered a variety of low-tech devices (e.g., CD players, MP3 players) that can be purchased for a range of prices and that can play customized audio recordings. The low-cost and low-tech nature of audio devices combined with the ease with which caregivers could use the devices, after the stories were created and recorded, suggested leveraging the auditory modality rather than the visual modality. These issues suggested further that a passive rather than a contingent stimulation strategy would enhance affordability when research findings are translated to daily rehabilitation and also provide more choices for sensory stimuli material that was familiar to the participant prior to injury (i.e., encoded into LTM), enabling us to further leverage principles of plasticity and abilities of the brain.

Since fMRI is an informative tool that we wanted to use to obtain mechanistic data, we examined feasibility of using fMRI to examine neural responses to visual, auditory, and temperature inputs in patients who were either comatose, VS, or MCS by contrasting a fixation cross projected on a screen with flashing checkerboards [69], preinjury music preferences with and without lyrics, hot and cold temperatures, as well as nonfamiliar and familiar voices relative to nonvocal sounds.

The visual modality was eliminated because not all patients in states of seriously impaired consciousness maintain eye-opening, and this would potentially become impossible to systematically control in an RCT. Temperature was eliminated as an intervention modality for two reasons. Since the biomedical device necessary to provide temperatures in a standardized manner that is also magnet compatible was not owned by every participating research site, fMRI examination of responses to passive temperature stimuli could become cost prohibitive in a clinical trial. Temperature was also eliminated because of constraints related to associating specific temperatures to preinjury/LTM memory traces (e.g., downhill snow skiing versus water skiing).

Given the constraints related to the visual and temperature modalities, the affordability and low-tech nature of auditory devices, and the theory and evidence previously summarized, we selected the auditory modality for our intervention.

\section{Illustration of Rationale for Developing and Examining FAST Protocol}

To demonstrate the effects of familiar auditory stimulation, this section provides findings from repeat functional imaging of persons without TBI listening to familiar and nonfamiliar voices orally reading a short story (Figure 1). The methods used to acquire and analyze the images in Figure 2 are provided in Figure 1. Figure 2 displays mean activation ( $p<0.001$ ) maps (T-maps) and the brain slice for each image is defined by the coordinates $(x, y$, and $z$ ), which are provided at the bottom of each image; a $z$-plane map is provided for reference.

The activation maps in Figures 2(a) and 2(b) demonstrate the activation to familiar voices orally reading a short story (FV Lang) and nonfamiliar voices orally reading the same story (NFV Lang). Figure 2(c) shows activation to nonfamiliar voices accounting for activation due to familiar voices. The familiar voice language map (Figure 2(d)) illustrates activation to familiar voices reading the story after accounting for/controlling for activation due to nonfamiliar voices. A direct comparison of Figures 2(c) and 2(d) indicates, as expected, differential (more) neural responses 


\section{fMRI Methods}

Subjects: No neurological history: 2 right-handed females (22 \& 29 yr) \& 2 right-handed males (34 \& 45 yr).

fMRI Block Design Protocol:

- Passive listening to set of auditory stimuli repeated 8 times; each set includes 6 types of audition \& each type lasts approximately $30 \mathrm{~s}$; randomized presentation order of auditory stimuli within each of 8 sets.

- Stimuli illustrated in Figure 2 contrast FV \& NFV, where NFV is gender matched to FV. Each subject listens to FV \& NFV orally reading this short story (FV Lang \& NFV Lang):

"A young man worked the night shift at the hospital, and every night his wife waited up for him. One night, he decided to treat his wife to a midnight snack. He stopped at an all-night diner and ordered a hot fudge sundae to go. As he waited for it, he saw a jar of dill pickles on the counter. When the proprietor came back with the ice cream, the young man ordered a pickle for himself. The proprietor put the sundae and the pickle in a box and said the total came to three dollars. The young man reached for his wallet, only to find he had left it at work. All he had was a dollar and fifty cents in change. The owner looked at the ice cream, then at the pickle, and smiled. He told the young man a dollar-fifty was enough."

Repeat Scans: 6 wk between repeat scans using identical protocols.

Image Acquisition: Whole brain, single-shot gradient echo planar imaging sensitive to contrast on 3T Siemens Trio whole body scanner with 12-channel head coil. For all scans (TR $=3 \mathrm{~s} ; \mathrm{TE}=40 \mathrm{~ms} ; \mathrm{FOV}=220 \mathrm{~mm}$ ), initial 6 volumes were discarded to allow gradient stabilization.

Image Processing \& Analyses: All functional images were corrected for slice timing \& realigned in SPM5 (http:// www.fil.ion.ucl.ac.uk/spm/). All structural images were normalized to MNI152 template \& then mean image was created. Whole brain analyses were computed using SPM5. Motion parameters were included as regressors. Whole brain activation was averaged for each individual stimulus. Fixed effect analysis was used to identify average responses for these contrasts: FV > 0, NFV > 0 (Figures 2(a) \& 2(b)), respectively), NFV > FV (Figure 2(c)), \& FV > NFV (Figure 2(d)). Since results are not corrected for multiple comparisons, significant activation is set at $p<0.001$. Random effect (second level) analysis was avoided considering small sample size (<10 subjects).

\section{Figure 1.}

Functional magnetic resonance imaging (fMRI) methods. FOV = field of view, FV = familiar voice, $\mathrm{MNI}=$ Montreal Neurological Institute, NFV = nonfamiliar voice, Lang = language, $T=$ Teslar, $T E=$ echo time, $T R=$ repetition time. Quote taken from Adamovich B, Henderson J. Scales of Cognitive Ability for Traumatic Brain Injury (SCATBI): Applied Symbolix, Inc; 1992.

to the familiar voice. More activation of the whole brain is seen for the familiar voice $(1.9 \%$ more activation and 51 more voxel clusters activated). In addition to a greater proportion of the brain activated, there is activation in more areas of the brain and these areas involve higher-level cortical processing areas. The significant $(p<0.001)$ activation in response to hearing familiar voices is illustrated (Figure 2(d)) in the prefrontal cortices, posterior cingulate cortex, parietal cortices (e.g., superior longitudinal fasiculus), temporal lobes bilaterally, and caudate nucleus.

In summary, the familiar voices relative to nonfamiliar elicit more neural responses, and this increased activation is located in higher-level cortical processing regions. These findings illustrate the effect of familiar auditory stimulation on the uninjured brain and provide further rationale to develop the FAST protocol and to examine it in the ongoing RCT.

\section{CONCLUSIONS: RANDOMIZED CLINICAL TRIAL-PURPOSE AND DESCRIPTION OF MAJOR DESIGN FEATURES}

The FAST protocol was developed, in part, because of the effects illustrated in the preceding section, and it is being examined in a double-blind RCT to determine whether or not this effect improves neurobehavioral functioning for persons in states of seriously impaired consciousness after severe TBI.

The RCT is a multisite study, in which subjects are recruited from Department of Veterans Affairs (VA) and private acute rehabilitation hospitals and from communities surrounding the geographic area of Chicago, Illinois, in the United States. Persons 18 years or older with severe TBI who have been comatose, VS, or MCS for at least 28 consecutive days and are within 1 year of injury are eligible for study enrollment. Persons are excluded if 
(a)

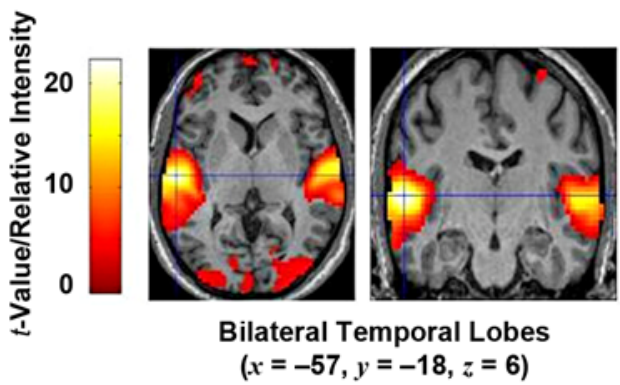

(b)

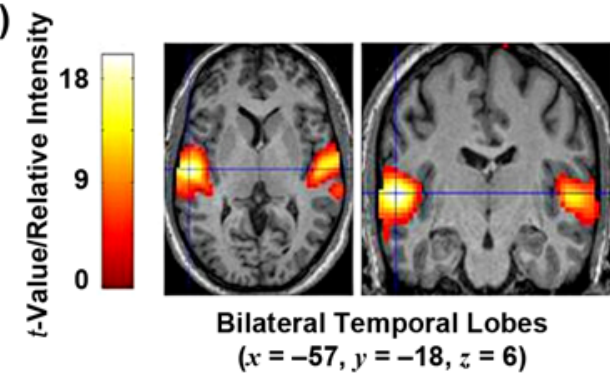

(d)

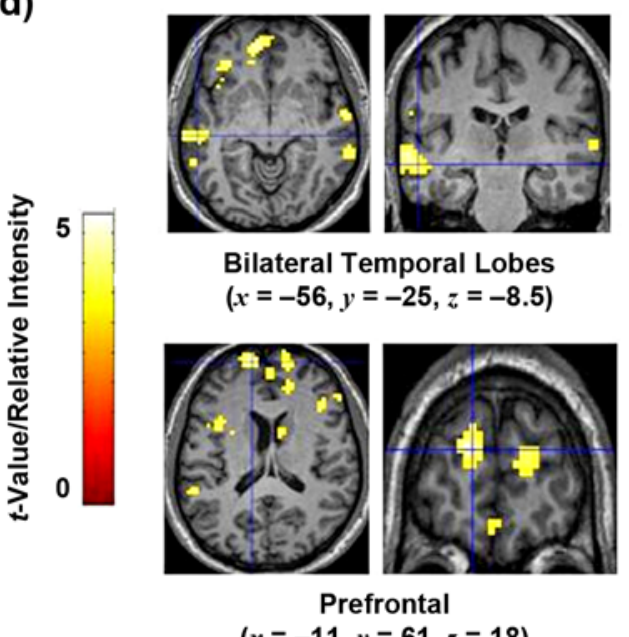

(e)

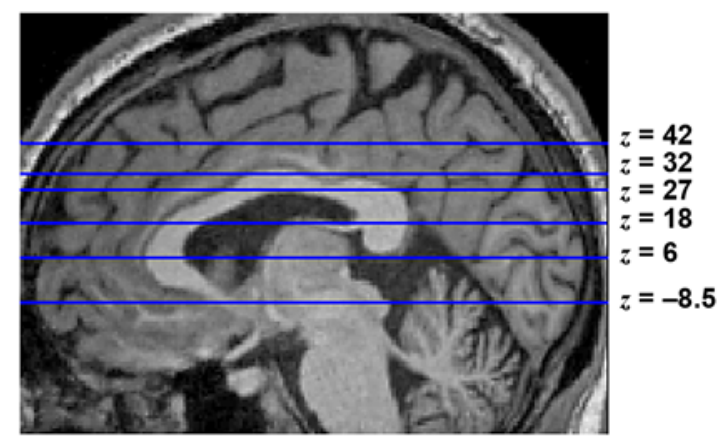

$z$ Reference Map

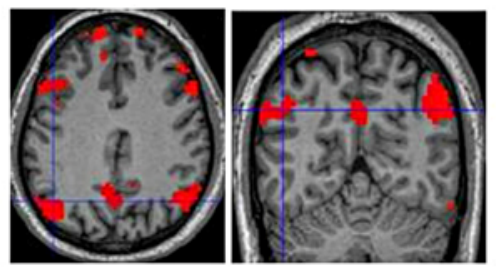

Parietal, Prefontal, Occipital $(x=-45, y=-67, z=32)$

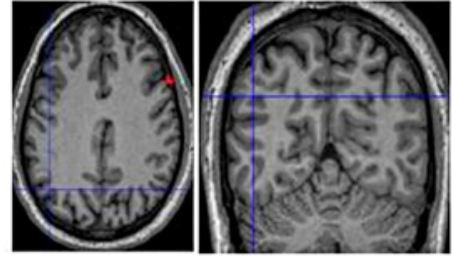

Right Parietal

$(x=-45, y=-67, z=32)$

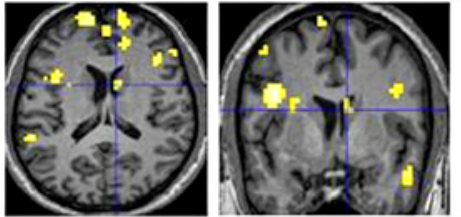

Caudate Nucleus

$(x=9, y=3, z=18)$
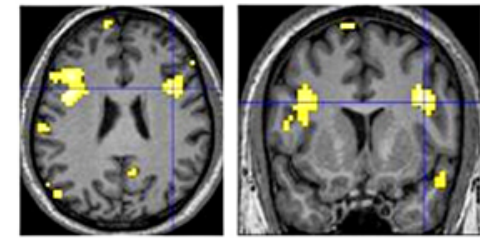

Superior Longitudinal Fasciculus I-III $(x=39, y=9, z=27)$ (c)
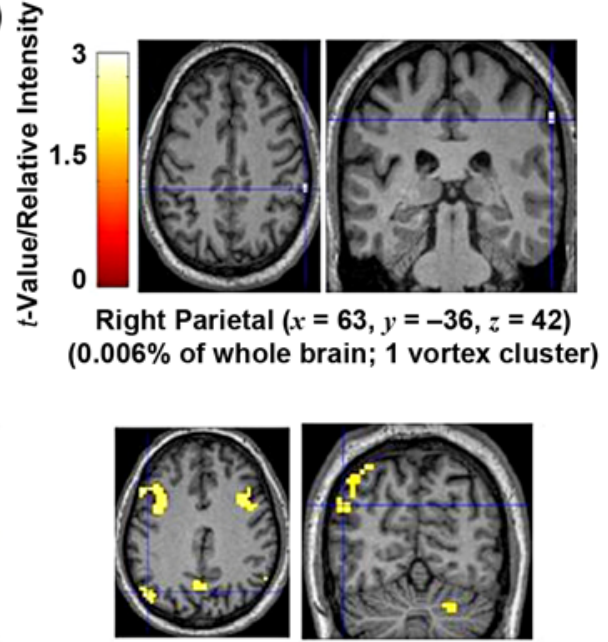

Left Parietal $(x=-47, y=-73, z=32)$

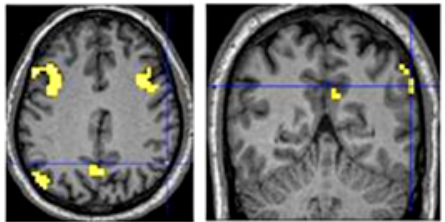

Right Parietal $(x=57, y=-60, z=33)$

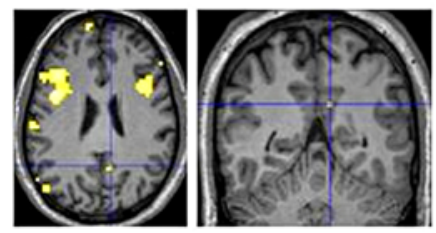

Posterior Cingulate $(x=8, y=-58, z=26)$

Figure 2.

(a) Activation to familiar voice (FV) reading story (FV > 0). (b) Activation to nonfamiliar voice (NFV) reading story (NFV > 0). (c) Differential activation to NFV reading story (NFV > FV). (d) Differential activation to FV reading story ( $2 \%$ of whole brain; 52 voxel clusters) (FV > NFV). (e) z-plane reference map. 
their primary brain injury is nontraumatic (e.g., vascular, anoxia) and if they experienced a seizure within the month preceding study enrollment. Additional exclusion criteria are shown in Figure 3.

Enrolled subjects are screened for adequate hearing prior to randomization to the experimental group (FAST) and sham control group; randomization to group is stratified within each site. Screening includes a brainstem auditory evoked potential (BAEP) and the auditory measure derived from the Disorders of Consciousness Scale (DOCS) [7072]. The presence of wave I [73] from the BAEP combined with the DOCS auditory measure [71,74] indicate, for the RCT, that the patient is sufficiently receiving sound.

Each participant receives a portable CD player at a preset volume to play the CDs. The experimental group receives the FAST protocol, and the sham group receives 1,680 minutes of silence provided in 10 minute daily segments at least 2 hours apart. For the sham group, each 10 -minute recording is a digital recording of silence. For both groups, the order of playing the CDs is randomized daily. Prior to obtaining baseline measures, each subject is titrated off of CNS stimulants. If a subject has been prescribed these stimulants as part of their usual care, they are then titrated back up to the stimulant dose after baseline measures are obtained. Since subjects are randomized to group, they can receive prescribed stimulants throughout the assigned intervention and usual medical rehabilitation care prescribed to them at time of study enrollment (e.g., range of motion, speech therapy two times a week).

Research staff obtaining measures of effect, nurses, clinical teams, family, significant others, and visitors are blinded to group assignment. Neurobehavioral testing is repeated weekly and it includes the DOCS [75]; the primary outcome is change in DOCS measures between baseline and primary endpoint. Neurophysiological measures, obtained at baseline and at primary endpoint, include BAEP tests, fMRI, resting state connectivity, and diffusion tensor imaging. Primary endpoint measures are obtained after all doses of the assigned intervention are provided. For ethical reasons and to maintain blinding, each participant receives the alternate intervention after the primary endpoint measures are obtained.

On a final note, it is important to mention that while the FAST protocol is theorized to extract consistent response across subjects, the only constants in the structure of the FAST protocol are duration, familiarity, and emotion. Each participant receives completely different auditory stimulation, with the differences spanning almost

\section{Patients are also excluded if they-}

- Have a brain-penetrating gunshot wound.

- Have a history of severe brain injury.

- Have contraindications to MRI (e.g., titanium in brain).

- Are ventilator dependent.

- Have cardiac contraindications.

- Have indications that sound is not being received.

\section{Figure 3.}

Exclusion criteria. $\mathrm{MRI}=$ magnetic resonance imaging .

all parameters of measurement of the structure of the auditory stimulation (e.g., speech rate, prosody). These parameters are included in the FAST protocol because we theorize that they will therapeutically affect how the injured brain processes and responds to the stimulation. The ongoing RCT, however, will not identify which parameter drives the brain's responses, but it will identify whether the brain responds and how this relates to changes in neurobehavioral functioning. Future research should be conducted to identify the parameters of the FAST protocol that promote behavioral gains.

\section{ACKNOWLEDGMENTS}

\section{Author Contributions:}

Study concept and design: T. L. Pape, J. M. Rosenow, S. McNamee, A. Guernon.

Acquisition of data: T. L. Pape, J. M. Rosenow, B. Harton, A. Guernon, T. Parrish, K. Froehlich, C. Burress, B. Weiss.

Analysis and interpretation of data: T. L. Pape, J. M. Rosenow, V. Patil, A. Guernon, T. Parrish, A. A. Herrold, X. Wang.

Drafting of manuscript: T. L. Pape, J. M. Rosenow, B. Harton, V. Patil, A. Guernon, T. Parrish, K. Froehlich, C. Burress, A. A. Herrold, $\mathrm{X}$. Wang.

Critical revision of manuscript for important intellectual content:

T. L. Pape, J. M. Roseneow, B. Harton, T. Parrish, X. Wang.

Obtained funding: T. L. Pape, J. M. Rosenow, S. McNamee, T. Parrish. Administrative, technical, or material support: B. Harton, V. Patil, T. Parrish, X. Wang, B. Weiss.

Study supervision: T. L. Pape, J. M. Rosenow, S. McNamee.

Financial Disclosures: The authors have declared that no competing interests exist.

Funding/Support: This material was based on work supported by VA Office of Research and Development, Rehabilitation Research and Development Service (grant B4591R and career development transition award B4949N); Northwestern University's Clinical and Translation Sciences Institute, which is supported by the National Center for Research Resources, National Institutes of Health (grant UL1RR025741); and The Nick Kot Charity for TBI (http://www.nkc4tbi.com). 
Institutional Review: The RCT described in this article has been approved by the human subjects institutional review boards for each participating site.

Participant Follow-Up: The authors plan to inform participants' legally authorized representatives of the publication of this article related to their study participation.

Additional Contributions: We wish to acknowledge and thank the study participants as well as their families, caregivers, and legally authorized representatives: without their participation, scientific and clinical knowledge could not be advanced. The research is also only possible with the cooperation of participating study sites and these include Edward Hines Jr VA Hospital, the Rehabilitation Institute of Chicago, and Hunter Holmes McGuire VA Medical Center. Substantial in-kind contributions from the Departments of Neurosurgery and Physical Medicine and Rehabilitation of Northwestern University Feinberg School of Medicine as well as Northwestern Memorial Hospital and Marianjoy Rehabilitation Hospital also make this research possible. Disclaimer: The views expressed in this article are those of the authors and do not necessarily reflect the position or policy of the VA or U.S. Government.

\section{REFERENCES}

1. Bushnik T, Hanks RA, Kreutzer J, Rosenthal M. Etiology of traumatic brain injury: characterization of differential outcomes up to 1 year postinjury. Arch Phys Med Rehabil. 2003;84(2):255-62. [PMID:12601658] http://dx.doi.org/10.1053/apmr.2003.50092

2. Summers CR, Ivins B, Schwab KA. Traumatic brain injury in the United States: an epidemiologic overview. Mt Sinai J Med. 2009;76(2):105-10. [PMID:19306375] http://dx.doi.org/10.1002/msj.20100

3. Nakase-Richardson R, Yablon SA, Sherer M, Nick TG, Evans CC. Emergence from minimally conscious state: insights from evaluation of posttraumatic confusion. Neurology. 2009; 73(14):1120-26. [PMID:19805728] http://dx.doi.org/10.1212/WNL.0b013e3181bacf34

4. Seel RT, Sherer M, Whyte J, Katz DI, Giacino JT, Rosenbaum AM, Hammond FM, Kalmar K, Pape TL, Zafonte R, Biester RC, Kaelin D, Kean J, Zasler N; American Congress of Rehabilitation Medicine, Brain Injury-Interdisciplinary Special Interest Group, Disorders of Consciousness Task Force. Assessment scales for disorders of consciousness: evidence-based recommendations for clinical practice and research. Arch Phys Med Rehabil. 2010;91(12):1795-1813. [PMID:21112421]

http://dx.doi.org/10.1016/j.apmr.2010.07.218

5. Giacino JT, Schnakers C, Rodriguez-Moreno D, Kalmar K, Schiff N, Hirsch J. Behavioral assessment in patients with disorders of consciousness: gold standard or fool's gold? Prog Brain Res. 2009;177:33-48. [PMID:19818893] http://dx.doi.org/10.1016/S0079-6123(09)17704-X
6. Overgaard M. How can we know if patients in coma, vegetative state or minimally conscious state are conscious? Prog Brain Res. 2009;177:11-19. [PMID:19818891] http://dx.doi.org/10.1016/S0079-6123(09)17702-6

7. Giacino JT, Ashwal S, Childs N, Cranford R, Jennett B, Katz DI, Kelly JP, Rosenberg JH, Whyte J, Zafonte RD, Zasler ND. The minimally conscious state: definition and diagnostic criteria. Neurology. 2002;58(3):349-53. [PMID:11839831] http://dx.doi.org/10.1212/WNL.58.3.349

8. Narayan RK, Michel ME, Ansell B, Baethmann A, Biegon A, Bracken MB, Bullock MR, Choi SC, Clifton GL, Contant CF, Coplin WM, Dietrich WD, Ghajar J, Grady SM, Grossman RG, Hall ED, Heetderks W, Hovda DA, Jallo J, Katz RL, Knoller N, Kochanek PM, Maas AI, Majde J, Marion DW, Marmarou A, Marshall LF, McIntosh TK, Miller E, Mohberg N, Muizelaar JP, Pitts LH, Quinn P, Riesenfeld G, Robertson CS, Strauss KI, Teasdale G, Temkin N, Tuma R, Wade C, Walker MD, Weinrich M, Whyte J, Wilberger J, Young AB, Yurkewicz L; The Clinical Trials in Head Injury Study Group. Clinical trials in head injury. J Neurotrauma. 2002;19(5):503-57. [PMID:12042091] http://dx.doi.org/10.1089/089771502753754037

9. NIH Consensus Development Panel on Rehabilitation of Persons With Traumatic Brain Injury. Consensus conference. Rehabilitation of persons with traumatic brain injury. JAMA. 1999;282(10):974-83. [PMID:10485684] http://dx.doi.org/10.1001/jama.282.10.974

10. Vanier M, Lamoureux J, Dutil E, Houde S. Clinical efficacy of stimulation programs aimed at reversing coma or vegetative state (VS) following traumatic brain injury. Acta Neurochir Suppl. 2002;79:53-57. [PMID:11974988] http://dx.doi.org/10.1007/978-3-7091-6105-0 12

11. Whyte J. Clinical trials in rehabilitation: what are the obstacles? Am J Phys Med Rehabil. 2003;82(10 Suppl): S16-21. [PMID:14502034] http://dx.doi.org/10.1097/01.PHM.0000086992.95121.C2

12. Giacino J. Sensory stimulation: theoretical perspectives and the evidence for effectiveness. NeuroRehabilitation. 1996;6: 69-78. http://dx.doi.org/10.1016/1053-8135(95)00149-2

13. Lancioni GE, Bosco A, Belardinelli MO, Singh NN, O'Reilly MF, Sigafoos J. An overview of intervention options for promoting adaptive behavior of persons with acquired brain injury and minimally conscious state. Res Dev Disabil. 2010;31(6):1121-34. [PMID:20663643] http://dx.doi.org/10.1016/j.ridd.2010.06.019

14. Kleim JA, Jones TA. Principles of experience-dependent neural plasticity: implications for rehabilitation after brain damage. J Speech Lang Hear Res. 2008;51(1):S225-39. [PMID:18230848] http://dx.doi.org/10.1044/1092-4388(2008/018) 
15. Ludlow CL, Hoit J, Kent R, Ramig LO, Shrivastav R, Strand E, Yorkston K, Sapienza CM. Translating principles of neural plasticity into research on speech motor control recovery and rehabilitation. J Speech Lang Hear Res. 2008; 51(1):S240-58. [PMID:18230849] http://dx.doi.org/10.1044/1092-4388(2008/019)

16. Hebb D. The organization of behavior. New York: Wiley; 1949.

17. Johansson BB. Brain plasticity and stroke rehabilitation. The Willis lecture. Stroke. 2000;31(1):223-30. [PMID:10625741] http://dx.doi.org/10.1161/01.STR.31.1.223

18. Spierer L, De Lucia M, Bernasconi F, Grivel J, Bourquin NM, Clarke S, Murray MM. Learning-induced plasticity in human audition: objects, time, and space. Hear Res. 2011; 271(1-2):88-102. [PMID:20430070] http://dx.doi.org/10.1016/j.heares.2010.03.086

19. Hubel DH, Wiesel TN. Binocular interaction in striate cortex of kittens reared with artificial squint. J Neurophysiol. 1965;28(6):1041-59. [PMID:5883731]

20. Andrews K. International Working Party on the Management of the Vegetative State: summary report. Brain Inj. 1996;10(11):797-806. [PMID:8905158] http://dx.doi.org/10.1080/026990596123918

21. Ansell BJ. Slow-to-recover brain-injured patients: rationale for treatment. J Speech Hear Res. 1991;34(5):1017-22. [PMID:1749232]

22. Nudo RJ, Jenkins WM, Merzenich MM. Repetitive microstimulation alters the cortical representation of movements in adult rats. Somatosens Mot Res. 1990;7(4):463-83. [PMID:2291378] http://dx.doi.org/10.3109/08990229009144720

23. Kleim JA, Barbay S, Nudo RJ. Functional reorganization of the rat motor cortex following motor skill learning. J Neurophysiol. 1998;80(6):3321-25. [PMID:9862925]

24. Pantev C, Herholz SC. Plasticity of the human auditory cortex related to musical training. Neurosci Biobehav Rev. 2011;35(10):2140-54. [PMID:21763342] http://dx.doi.org/10.1016/j.neubiorev.2011.06.010

25. Kleim JA, Hogg TM, VandenBerg PM, Cooper NR, Bruneau R, Remple M. Cortical synaptogenesis and motor map reorganization occur during late, but not early, phase of motor skill learning. J Neurosci. 2004;24(3):628-33. [PMID:14736848] http://dx.doi.org/10.1523/JNEUROSCI.3440-03.2004

26. Meinzer M, Elbert T, Wienbruch C, Djundja D, Barthel G, Rockstroh B. Intensive language training enhances brain plasticity in chronic aphasia. BMC Biol. 2004;2. [PMID:15028118]

27. Pulvermüller F, Neininger B, Elbert T, Mohr B, Rockstroh B, Koebbel P, Taub E. Constraint-induced therapy of chronic aphasia after stroke. Stroke. 2001;32(7):1621-26.

\section{[PMID:11441210]}

http://dx.doi.org/10.1161/01.STR.32.7.1621

28. Gabriel DA, Kamen G, Frost G. Neural adaptations to resistive exercise: mechanisms and recommendations for training practices. Sports Med. 2006;36(2):133-49.

[PMID:16464122]

http://dx.doi.org/10.2165/00007256-200636020-00004

29. May PJ, McHaffie JG, Stanford TR, Jiang H, Costello MG, Coizet V, Hayes LM, Haber SN, Redgrave P. Tectonigral projections in the primate: a pathway for pre-attentive sensory input to midbrain dopaminergic neurons. Eur J Neurosci. 2009;29(3):575-87. [PMID:19175405] http://dx.doi.org/10.1111/j.1460-9568.2008.06596.x

30. Schwender D, Kaiser A, Klasing S, Peter K, Pöppel E. Midlatency auditory evoked potentials and explicit and implicit memory in patients undergoing cardiac surgery. Anesthesiology. 1994;80(3):493-501. [PMID:8141445] http://dx.doi.org/10.1097/00000542-199403000-00004

31. Laureys S, Goldman S, Phillips C, Van Bogaert P, Aerts J, Luxen A, Franck G, Maquet P. Impaired effective cortical connectivity in vegetative state: preliminary investigation using PET. Neuroimage. 1999;9(4):377-82.

[PMID:10191166]

http://dx.doi.org/10.1006/nimg.1998.0414

32. Marshall JC, Halligan PW. Blindsight and insight in visuospatial neglect. Nature. 1988;336(6201):766-67.

[PMID:3205302]

http://dx.doi.org/10.1038/336766a0

33. Jones R, Hux K, Morton-Anderson KA, Knepper L. Auditory stimulation effect on a comatose survivor of traumatic brain injury. Arch Phys Med Rehabil. 1994;75(2):164-71. [PMID:8311672]

34. Laureys S, Faymonville ME, Degueldre C, Fiore GD, Damas P, Lambermont B, Janssens N, Aerts J, Franck G, Luxen A, Moonen G, Lamy M, Maquet P. Auditory processing in the vegetative state. Brain. 2000;123(Pt 8):1589-1601. [PMID:10908189] http://dx.doi.org/10.1093/brain/123.8.1589

35. Menon DK, Owen AM, Williams EJ, Minhas PS, Allen CM, Boniface SJ, Pickard JD; Wolfson Brain Imaging Centre Team. Cortical processing in persistent vegetative state. Lancet. 1998;352(9123):200. [PMID:9683212] http://dx.doi.org/10.1016/S0140-6736(05)77805-3

36. Moritz CH, Rowley HA, Haughton VM, Swartz KR, Jones J, Badie B. Functional MR imaging assessment of a nonresponsive brain injured patient. Magn Reson Imaging. 2001;19(8):1129-32. [PMID:11711238] http://dx.doi.org/10.1016/S0730-725X(01)00432-5

37. Näätänen R, Gaillard AW, Mäntysalo S. Early selectiveattention effect on evoked potential reinterpreted. Acta Psychol (Amst). 1978;42(4):313-29. [PMID:685709] http://dx.doi.org/10.1016/0001-6918(78)90006-9 
38. Garrido MI, Kilner JM, Stephan KE, Friston KJ. The mismatch negativity: a review of underlying mechanisms. Clin Neurophysiol. 2009;120(3):453-63. [PMID:19181570] http://dx.doi.org/10.1016/j.clinph.2008.11.029

39. Wijnen VJ, van Boxtel GJ, Eilander HJ, de Gelder B. Mismatch negativity predicts recovery from the vegetative state. Clin Neurophysiol. 2007;118(3):597-605.

[PMID:17239656]

http://dx.doi.org/10.1016/j.clinph.2006.11.020

40. Schröger E, Näätänen R, Paavilainen P. Event-related potentials reveal how non-attended complex sound patterns are represented by the human brain. Neurosci Lett. 1992; 146(2):183-86. [PMID:1491786] http://dx.doi.org/10.1016/0304-3940(92)90073-G

41. Garagnani M, Pulvermüller F. From sounds to words: a neurocomputational model of adaptation, inhibition and memory processes in auditory change detection. Neuroimage. 2011;54(1):170-81. [PMID:20728545] http://dx.doi.org/10.1016/j.neuroimage.2010.08.031

42. Kane NM, Curry SH, Butler SR, Cummins BH. Electrophysiological indicator of awakening from coma. Lancet. 1993;341(8846):688. [PMID:8095587]

http://dx.doi.org/10.1016/0140-6736(93)90453-N

43. Qin P, Di H, Yan X, Yu S, Yu D, Laureys S, Weng X. Mismatch negativity to the patient's own name in chronic disorders of consciousness. Neurosci Lett. 2008;448(1):24-28. [PMID:18938213] http://dx.doi.org/10.1016/j.neulet.2008.10.029

44. Näätänen R, Tervaniemi M, Sussman E, Paavilainen P, Winkler I. "Primitive intelligence" in the auditory cortex. Trends Neurosci. 2001;24(5):283-88. [PMID:11311381] http://dx.doi.org/10.1016/S0166-2236(00)01790-2

45. Jääskeläinen IP, Ahveninen J, Bonmassar G, Dale AM, Ilmoniemi RJ, Levänen S, Lin FH, May P, Melcher J, Stufflebeam S, Tiitinen H, Belliveau JW. Human posterior auditory cortex gates novel sounds to consciousness. Proc Natl Acad Sci USA. 2004;101(17):6809-14. [PMID:15096618] http://dx.doi.org/10.1073/pnas.0303760101

46. Näätänen R. The role of attention in auditory information processing as revealed by event-related potentials and other brain measures of cognitive function. Behav Brain Sci. 1990; 13:201-33. http://dx.doi.org/10.1017/S0140525X00078407

47. Imaizumi S, Mori K, Kiritani S, Kawashima R, Sugiura M, Fukuda H, Itoh K, Kato T, Nakamura A, Hatano K, Kojima S, Nakamura K. Vocal identification of speaker and emotion activates different brain regions. Neuroreport. 1997; 8(12):2809-12. [PMID:9295122] http://dx.doi.org/10.1097/00001756-199708180-00031

48. Von Kriegstein K, Kleinschmidt A, Sterzer P, Giraud AL. Interaction of face and voice areas during speaker recognition. J Cogn Neurosci. 2005;17(3):367-76.

\section{[PMID:15813998]}

ttp://dx.doi.org/10.1162/0898929053279577

49. Binder JR, Frost JA, Hammeke TA, Bellgowan PS, Springer JA, Kaufman JN, Possing ET. Human temporal lobe activation by speech and nonspeech sounds. Cereb Cortex. 2000;10(5):512-28. [PMID:10847601]

http://dx.doi.org/10.1093/cercor/10.5.512

50. Zatorre RJ, Meyer E, Gjedde A, Evans AC. PET studies of phonetic processing of speech: review, replication, and reanalysis. Cereb Cortex. 1996;6(1):21-30.

[PMID:8670635]

http://dx.doi.org/10.1093/cercor/6.1.21

51. Gutzmann H, Kühl KP. Emotion control and cerebellar atrophy in senile dementia. Arch Gerontol Geriatr. 1987; 6(1):61-71. [PMID:2884937] http://dx.doi.org/10.1016/0167-4943(87)90039-2

52. National Institutes of Health. Consensus development conference on brain injury rehabilitation [Internet]. Bethesda (MD): National Institutes of Health; 1998. Available from: http://consensus.nih.gov/1998/ 1998TraumaticBrainInjury109html.htm

53. Nakamura K, Kawashima R, Sugiura M, Kato T, Nakamura A, Hatano K, Nagumo S, Kubota K, Fukuda H, Ito K, Kojima S. Neural substrates for recognition of familiar voices: a PET study. Neuropsychologia. 2001;39(10): 1047-54. [PMID:11440757] http://dx.doi.org/10.1016/S0028-3932(01)00037-9

54. Shah NJ, Marshall JC, Zafiris O, Schwab A, Zilles K, Markowitsch HJ, Fink GR. The neural correlates of person familiarity. A functional magnetic resonance imaging study with clinical implications. Brain. 2001;124(Pt 4):804-15. [PMID:11287379] http://dx.doi.org/10.1093/brain/124.4.804

55. Van Lancker DR, Cummings JL, Kreiman J, Dobkin BH. Phonagnosia: a dissociation between familiar and unfamiliar voices. Cortex. 1988;24(2):195-209. [PMID:3416603]

56. Van Lancker DR, Kreiman J, Cummings J. Voice perception deficits: neuroanatomical correlates of phonagnosia. J Clin Exp Neuropsychol. 1989;11(5):665-74. [PMID:2808656] http://dx.doi.org/10.1080/01688638908400923

57. Kurtz D, Trapp C, Kieny C, et al. Study of recovery and the post-anesthetic period. Rev Electroencephal Neurophys Clin. 1977;7:62-69.

http://dx.doi.org/10.1016/S0370-4475(77)80036-1

58. Signorino M, D’Acunto S, Angeleri F, Pietropaoli P. Eliciting P300 in comatose patients. Lancet. 1995;345(8944): 255-56. [PMID:7823734] http://dx.doi.org/10.1016/S0140-6736(95)90252-X

59. Perrin F, Schnakers C, Schabus M, Degueldre C, Goldman S, Brédart S, Faymonville ME, Lamy M, Moonen G, Luxen A, Maquet P, Laureys S. Brain response to one's own name in vegetative state, minimally conscious state, and locked-in 
syndrome. Arch Neurol. 2006;63(4):562-69. [PMID:16606770]

http://dx.doi.org/10.1001/archneur.63.4.562

60. Gorno-Tempini ML, Price CJ, Josephs O, Vandenberghe R, Cappa SF, Kapur N, Frackowiak RS. The neural systems sustaining face and proper-name processing. Brain. 1998; 121(Pt 11):2103-18. [PMID:9827770] http://dx.doi.org/10.1093/brain/121.11.2103

61. Moray N. Attention in dichotic listening: Affective cues and the influence of instructions. Q J Exp Psychol. 1959; 11:56-60. http://dx.doi.org/10.1080/17470215908416289

62. Proverbio AM, Lilli S, Semenza C, Zani A. ERP indexes of functional differences in brain activation during proper and common names retrieval. Neuropsychologia. 2001;39(8): 815-27. [PMID:11369405] http://dx.doi.org/10.1016/S0028-3932(01)00003-3

63. Yasuda Y, Nakamura T, Beckman B. Brain processing of proper names. Aphasiology. 2000;14:1067-89. http://dx.doi.org/10.1080/02687030050174638

64. Di HB, Yu SM, Weng XC, Laureys S, Yu D, Li JQ, Qin PM, Zhu YH, Zhang SZ, Chen YZ. Cerebral response to patient's own name in the vegetative and minimally conscious states. Neurology. 2007;68(12):895-99.

[PMID:17372124]

http://dx.doi.org/10.1212/01.wnl.0000258544.79024.d0

65. Kartje GL, Schwab ME. Axonal growth in the adult mammalian nervous system: regeneration and compensatory plasticity. In: Siegel G, Albers RW, Brady S, Price D, editors. Basic neurochemistry: molecular, cellular, and medical aspects. 7th ed. Burlington (MA): Elsevier; 2006. p. 517-28.

66. Cleeremans A, Destrebecqz A, Boyer M. Implicit learning: news from the front. Trends Cogn Sci. 1998;2(10):406-16. [PMID:21227256] http://dx.doi.org/10.1016/S1364-6613(98)01232-7

67. Von Kriegstein K, Eger E, Kleinschmidt A, Giraud AL. Modulation of neural responses to speech by directing attention to voices or verbal content. Brain Res Cogn Brain Res. 2003;17(1):48-55. [PMID:12763191] http://dx.doi.org/10.1016/S0926-6410(03)00079-X

68. Buckner RL, Goodman J, Burock M, Rotte M, Koutstaal W, Schacter D, Rosen B, Dale AM. Functional-anatomic correlates of object priming in humans revealed by rapid presentation event-related fMRI. Neuron. 1998;20(2):285-96. [PMID:9491989] http://dx.doi.org/10.1016/S0896-6273(00)80456-0

69. Pape T, Gitelman D, Parrish T, Senno R, Kelly J, Weiner B. Measurement of cerebral activity in male participant four and six years post severe brain injury. Arch Phys Med Rehabil. 2001;82(9):1291-1346.

70. Pape TL, Heinemann AW, Kelly JP, Hurder AG, Lundgren S. A measure of neurobehavioral functioning after coma. Part I: Theory, reliability, and validity of Disorders of Con- sciousness Scale. J Rehabil Res Dev. 2005;42(1):1-17. [PMID:15742245]

http://dx.doi.org/10.1682/JRRD.2004.03.0032

71. Pape TL, Senno RG, Guernon A, Kelly JP. A measure of neurobehavioral functioning after coma. Part II: Clinical and scientific implementation. J Rehabil Res Dev. 2005; 42(1):19-27. [PMID:15742246] http://dx.doi.org/10.1682/JRRD.2004.03.0033

72. Pape TL, Tang C, Guernon A, Lundgren S, Blahnik M, Wei Y, Querubin M, Zollman F, Soneru I. Predictive value of the Disorders of Consciousness Scale (DOCS). Phys Med Rehabil. 2009;1(2):152-61. [PMID:19627889] http://dx.doi.org/10.1016/j.pmrj.2008.11.002

73. Chiappa KH, Hill RA. Brain stem auditory evoked potentials: interpretation. In: Chiappa KH, editor. Evoked potentials in clinical medicine. 3rd ed. Philadelphia (PA): LippincottRaven; 1997. p. 199-250.

74. Pape T. Acute neurorehabilitation for disorders of consciousness. In: Selzer M, Miller R, Cohen L, Clarke S, Kwakke G, editors. Neural Repair \& Rehabilitation. 2nd ed. Cambridge (UK): Cambridge University Press; 2012.

75. Pape T, Lundgren S, Guernon A, Kelly J, Heinemann A. Disorders of Consciousness Scale (DOCS): administration manual [Internet]. Washington (DC): Department of Veterans Affairs; 2011. Available from:

http://www.queri.research.va.gov/ptbri/docs training/ manual 2011.pdf

Submitted for publication August 31, 2011. Accepted in revised form April 30, 2012.

This article and any supplementary material should be cited as follows:

Pape TL, Rosenow JM, Harton B, Patil V, Guernon A, Parrish T, Froehlich K, Burress C, McNamee S, Herrold AA, Weiss B, Wang X. Preliminary framework for Familiar Auditory Sensory Training (FAST) provided during coma recovery. J Rehabil Res Dev. 2012;49(7):1137-52.

http://dx.doi.org/10.1682/JRRD.2011.08.0154

ResearcherID: Theresa Louise-Bender Pape, DrPH, MA, CCC-SLP/L: G-3591-2012

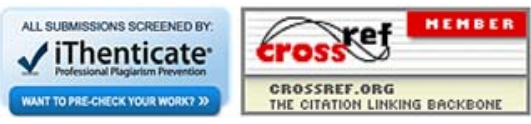

